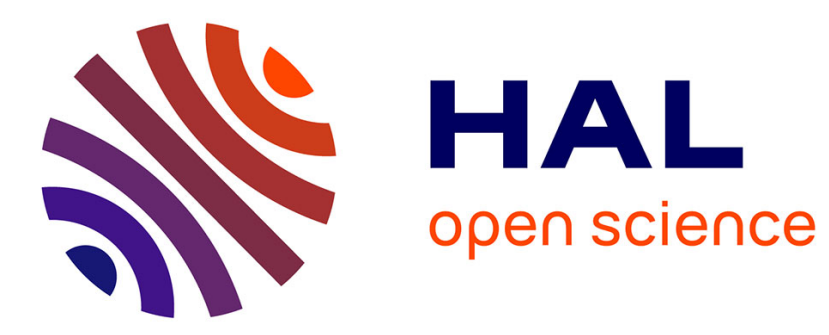

\title{
Energy Consumption and Performance of IEEE 802.15.4e TSCH and DSME
}

Iacob Juc, Olivier Alphand, Roberto Guizzetti, Michel Favre, Andrzej Duda

\section{To cite this version:}

Iacob Juc, Olivier Alphand, Roberto Guizzetti, Michel Favre, Andrzej Duda. Energy Consumption and Performance of IEEE 802.15.4e TSCH and DSME. Proc. of the IEEE Wireless Communications and Networking Conference (WCNC), IEEE, Apr 2016, Doha, Qatar. hal-01287512

\section{HAL Id: hal-01287512 \\ https://hal.science/hal-01287512}

Submitted on 13 Mar 2016

HAL is a multi-disciplinary open access archive for the deposit and dissemination of scientific research documents, whether they are published or not. The documents may come from teaching and research institutions in France or abroad, or from public or private research centers.
L'archive ouverte pluridisciplinaire HAL, est destinée au dépôt et à la diffusion de documents scientifiques de niveau recherche, publiés ou non, émanant des établissements d'enseignement et de recherche français ou étrangers, des laboratoires publics ou privés. 


\title{
Energy Consumption and Performance of IEEE 802.15.4e TSCH and DSME
}

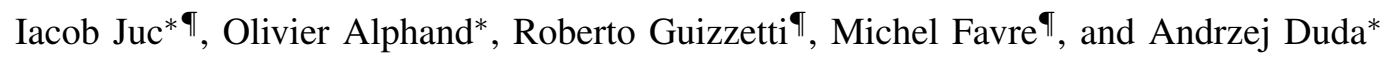 \\ *Grenoble Alps University, Grenoble Institute of Technology, Grenoble Informatics Laboratory, Grenoble, France \\ ISTMicroelectronics, Crolles, France \\ Email: \{firstname.lastname\}@imag.fr, \{firstname.lastname\}@ st.com
}

\begin{abstract}
The recent IEEE 802.15.4e standard has introduced two interesting modes of operation: Time Slotted Channel Hopping (TSCH) and Deterministic and Synchronous Multichannel Extension (DSME). Both provide a mix of time and frequency division to improve the performance of the previously available synchronized MAC mode (beacon-enabled 802.15.4). In this paper, we compare the performance of DSME and TSCH with respect to the energy consumption, throughput, and delay through an analysis of their respective ways of operation. We use an energy consumption model coming from our previous experience on the design of recent energy harvesting motes for the GreenNet platform.

Our results show that DSME performs slightly better in terms of the energy consumption spent in data transfers. Both protocols exhibit similar delays for a given duty cycle, nevertheless, TSCH obtains shorter delay and higher throughput for low duty cycles. For higher duty cycles, TSCH results in lower throughput-for applications that send little data, the fixed slot configuration of TSCH results in wasted bandwidth. DSME can allocate shorter slots, which is beneficial for applications that transmit short packets.
\end{abstract}

Index Terms -802.15.4e, TSCH, DSME, channel hopping, energy efficiency, WSN, LLN

\section{INTRODUCTION}

The IEEE 802.15.4 standard [1] published in 2003, 2006, and 2011 defined the PHY and MAC layers with two operating modes (non-beacon enabled and beacon-enabled) for low-power sensor and actuator networks. In 2012, the 802.15.4e revision [2] introduced several new MAC layer modes:

- DSME (Deterministic and Synchronous Multi-channel Extension) for deterministic latency and scalability requirements,

- TSCH (Time-Slotted Channel Hopping) for high throughput requirements, bounded latency, and high reliability.

- LLDN (Low Latency Deterministic Network) for high reliability and low latency,

- RFID Blink (Radio Frequency Identification) for item and people identification, location, and tracking,

- AMCA (Asynchronous Multi-Channel Adaptation) for infrastructure monitoring networks,
DSME is an extension of the beacon-enabled mode for better utilization of inactive periods. It retains the principle of periodic beacons of the beacon-enabled mode and takes advantage of channel diversity through channel hopping: devices switch channels according to a predefined sequence for each communication in a reserved time slot. Devices under DSME can also use channel adaptation: they can allocate reserved slots on separate channels, the channel for each slot not changing in time.

TSCH also benefits from channel hopping, but devices do not use beacons for synchronization (except for advertising network information). They rather operate according to a common shared schedule that determines which device may transmit frames on a given channel and during a given time slot. To follow the schedule, devices need to be synchronized, so nodes can benefit from the deterministic behavior and some level of the required quality of service resulting from the schedule allocation.

TSCH has attracted considerable attention from the Wireless Sensor Network community with the establishment of the IETF 6TiSCH Working Group [?] whose goal is to define the operation of Low-power and Lossy Networks (LLN) under RPL routing (Routing Protocol for Low-power and lossy networks) [3] over TSCH.

DSME and TSCH are the only new modes that support peer-to-peer and multi-hop topologies. In this paper, we compare the performance of the DSME and TSCH protocols with respect to the energy consumption, throughput, and delay through an analysis of their respective ways of operation. We assume two roles of nodes in the test scenario: a leaf node that generates sensor data and a router node that forwards the data in a multihop way to the sink.

From a practical point of view, extending existing beaconenabled 802.15.4 implementations to offer DSME would be easier than implementing TSCH. The comparison was partly motivated by the need to find out which of the two protocols better fits the GreenNet platform [4], previously running a beacon-enabled 802.15.4 protocol stack.

We use an energy consumption model coming from the experimental experience on the design of recent energy 
harvesting motes [4] and analyze how two nodes perform when exchanging packets under two protocols. Under our assumptions, we consider that a two-node scenario provides sufficient insight on the functioning of the protocols. Both protocols provide multi-channel operation, thus mitigating multi-path fading. By conveniently configuring them, we highlight their similarities and simplify the test scenario. Our results show that DSME performs slightly better in terms of the energy consumption spent in data transfers. Both protocols exhibit similar delays for a given duty cycle, nevertheless, TSCH obtains shorter delay and higher throughput for low duty cycles. For higher duty cycles, TSCH results in lower throughput-for applications that send little data, the fixed slot configuration of TSCH results in wasted bandwidth. DSME can allocate shorter slots, which is beneficial for applications that transmit short packets.

We start the paper with a presentation of DSME and TSCH in Section II. In Section III, we discuss parameter configurations that we adopt for the protocols. Then, in Section IV, we present the energy consumption model and the estimated energy costs for the protocols. Then, we compare the protocols in terms of throughput and delay. Section V discusses related work and Section VI presents conclusions and the future work.

\section{BACKGROUND ON DSME AND TSCH}

\section{A. DSME}

As an extension of the 802.15.4 beacon-enabled mode [1], DSME inherits some of its features such as beacons that synchronize data transfers, the basic superframe structure with time slots, and two types of devices: Full-Function Devices (FFDs) and Reduced-Function Devices (RFDs) [2]. FFD devices transmit beacons and act as coordinators while RFD only wake up at beacons to transmit data. Intermediary nodes act as routers to forward packets-they act as RFD devices towards their coordinators and as FFD towards their child nodes.
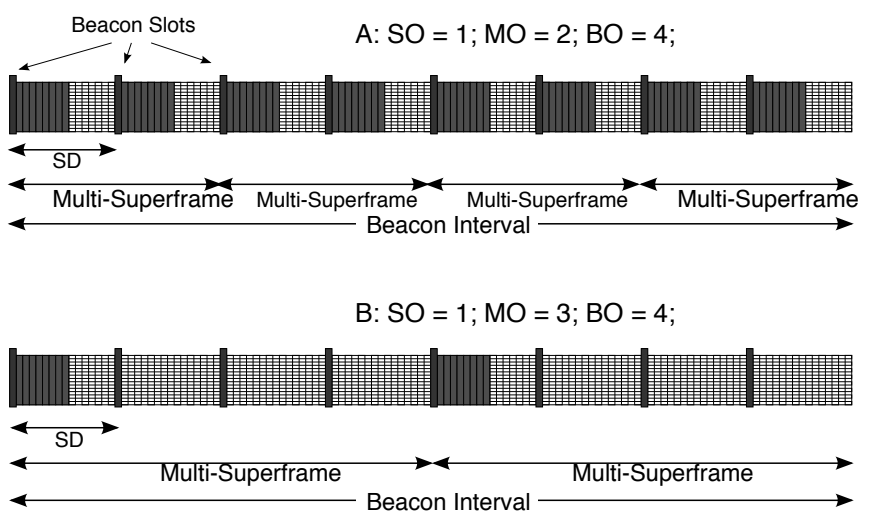

Figure 1. Example of DSME multi-superframes for different parameters: A) normal mode, B) CAP Reduction enabled.
DSME extends the 802.15.4 beacon-enabled superframe structure with the notion of a multi-superframe (cf. Fig.1):

- a superframe is composed of 16 slots of aBaseSlotLength $=960 * 2^{S O} \mu$ s and the Superframe Duration is $S D=$ aBaseSuperframeDuration $* 2^{S O}$, where $S O$ is the Superframe Order,

- $2^{(M O-S O)}$ superframes are grouped in a multisuperframe, where $M O$ is the Multi-superframe Order, - $2^{(B O-M O)}$ multi-superframes are contained in a Beacon Interval $B I=$ aBaseSuperframeDuration $* 2^{B O}$, where $B O$ is the Beacon Order. The Beacon Interval contains $2^{(B O-S O)}$ superframes.

In DSME, the Contention Access Period (CAP) is reduced to 9 slots (grey slots in the figure) and the remaining 7 slots are reserved for allocating Guaranteed Time Slots (GTS). Slot 0 is always reserved for Enhanced Beacons emission. In contrast with beacon-enabled 802.15.4, DSME can allocate GTS slots to any device in any GTS slot inside a multisuperframe (Fig. 1). By varying $M O$, we can adjust the repetition period of GTS slots inside the Beacon Interval.

Enhanced Beacons contain Information Elements (rather than a superframe specification) to describe various properties of the network, such as a bitmap to indicate the allocation of superframes inside the Beacon Interval. This mechanism for superframe allocation solves the problem of the multi-hop network construction in 802.15.4 beacon-enabled networks [5]. The maximum number of routing devices, including the PAN Coordinator is given by MaxRouter $=2^{(B O-S O)}$, the number of Superframes in the Beacon Interval.

DSME also introduces CAP Reduction: with CAP Reduction, only the first superframe in a multi-superframe has the CAP period and the CAP slots for the remaining superframes are available for GTS allocation. This mechanism reduces the energy consumption, since nodes do not need to wake up for the unused CAPs and the extra GTS slots allow for a better distribution of bandwidth. With CAP Reduction enabled (cf. Fig. 1-B), nodes can communicate with their coordinator in the remaining CAP.

During CAP, nodes proceed in four steps:

- beacon transmission,

- coordinator broadcast transmission,

- coordinator unicast transmission,

- device unicast transmission to coordinator.

As in beacon-enabled 802.15.4, coordinators announce frames pending for devices in beacons. To retrieve a data frame, a device polls the coordinator with a Data Request and the coordinator sends the corresponding data frame. Nodes transmit both Data Requests and data frames using the slotted CSMA/CA algorithm. The frames are acknowledged by ACK frames. 
of each protocol. They both provide mechanisms to reserve communication slots between pairs of nodes, the slots being grouped in superframe structures that repeat periodically. We shall propose configurations for the two protocols so that the resulting superframes are similar. We configure both protocols for a default check interval $T$ of $1 \mathrm{~s}$. Thus, by the 


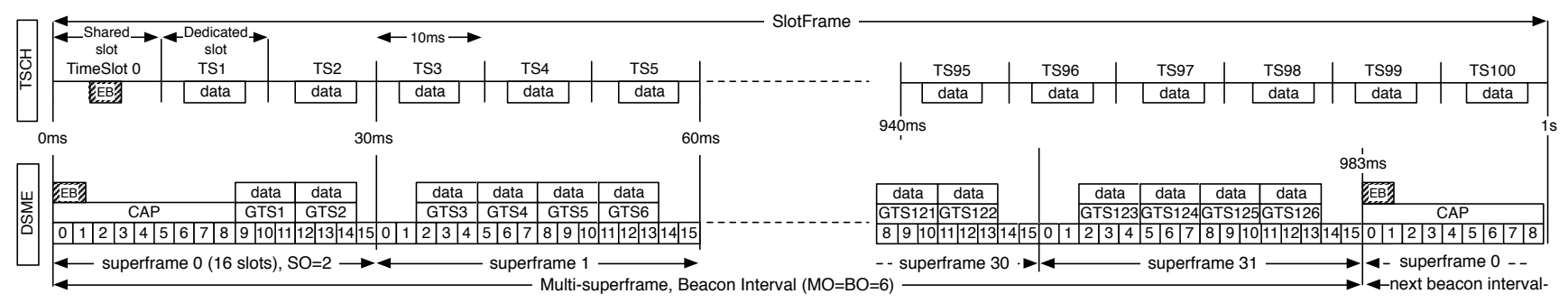

Figure 3. 6TiSCH minimal configuration: TSCH slotframe vs. DSME beacon interval

addresses, and no security). In the worst case, if using long addresses, the header is 23 byte long without security and 37 byte long with security [1]. We consider the most common case of the header without security.

We assume that applications generate packets that do not require 6LoWPAN fragmentation. In the case of fragmentation, the overhead added by 6LoWPAN would be 7 bytes, which would sum up to 18 bytes with the MAC header. The minimal CoAP [11] packet size is 4 bytes (the header without payload).

We consider three packet sizes: 30, 60 and 90 bytes and we take into account Enhanced ACK frames used by TSCH and described in Table II. DSME uses 5 byte long ACK frames as shown in Table III.

Table II

ENHANCED ACK FRAME

\begin{tabular}{|c|c|c|c|c|c|c|}
\hline FCF & Seq Number & Src Addr & Dest Addr & Sync IE & FCS & total \\
\hline 2 & 1 & 2 & 2 & 4 & 2 & $\mathbf{1 3}$ \\
\hline
\end{tabular}

Table III

ACK FRAME

\begin{tabular}{|c|c|c|c|}
\hline FCF & Seq Number & FCS & total \\
\hline 2 & 1 & 2 & $\mathbf{5}$ \\
\hline
\end{tabular}

The chosen values for TSCH slots are long enough to accommodate all packet sizes. DSME GTS of $6 \mathrm{~ms}$ duration can also accommodate a data exchange, including the ACK, with a maximal size packet.

\section{B. Communication Patterns}

We consider frame exchanges in both protocols that follow the patterns shown in Fig. 4. We adopt the parameters of the TSCH slot according to the $6 \mathrm{TiSCH}$ minimal configuration draft [10]. Inside a TSCH slot, nodes delay a transmission with respect to the beginning of the slot by an offset and the guard time defined in the TSCH timeslot structure. The default $t s T x O f f s e t$ is $2120 \mu$ s and the default guard time is $2000 \mu$ s. We consider that on the average, the receiver listens

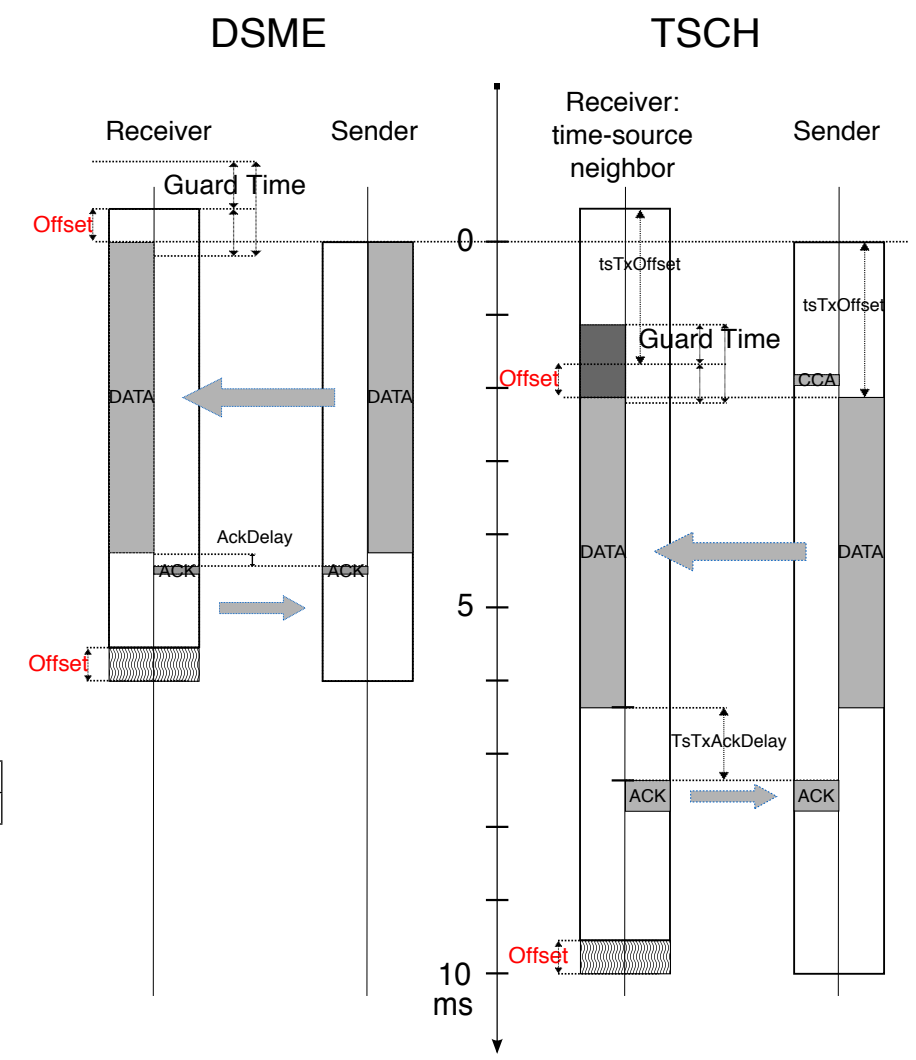

Figure 4. Data exchange patterns inside DSME GTS and TSCH shared slots.

for the half of the guard time. Clear Channel Assessment (CCA) is used in TSCH before a packet transmission, to prevent nodes from transmitting on a busy channel. CCA is optional, but we have chosen to take it into account. After CCA, the radio needs $192 \mu$ s to switch from Rx to Tx mode. Note that CCA is useful when several networks coexist, otherwise, CCA cannot detect and prevent multiple transmissions inside the same slot if the transmissions are synchronized.

In DSME, the transmission may be aligned with the beginning of a GTS slot. The ACK frame is transmitted 
$192 \mu$ s after the end of the received frame. The guard time is implementation dependent: when using a 10 ppm oscillator on the MS1.0 platform (GreenNet Project) and a Beacon Interval of 1 second, the drift should be less than $10 \mu \mathrm{s}$, while a radio symbol is $16 \mu$ s long. The guard time can be adjusted locally by each node, based on the interval between synchronizations, for example, doubled if a node missed a beacon.

\section{ENERGY CONSUMPTION COMPARISONS}

In this section, we first discuss the energy consumption model used for comparing energy consumption and then present the results of these comparisons.

\section{A. Energy Consumption Model}

We follow the recent work of Vilajosana et al. who proposed a simple energy consumption model for $\mathrm{TSCH}$ networks [12]. We also build on our previous work in which we have performed extensive evaluations of the energy consumption in GreenNet, an energy harvesting IP-enabled wireless sensor network [4]. We adopt the definition of a consumption profile as a sum of energy consumption contributions from each running peripheral. The radio and the micro-controller are the most energy consuming devices, thus we can obtain the total energy consumption by summing the time they are $\mathrm{ON}$ and considering how much power (or current intensity) they require in that state. Note that the radio consumes almost the same energy in reception as in transmission. Table IV presents the current intensity drawn in different states for various types of motes (data sources: MS1.0 [4], OpenMoteSTM, GINA [12]).

Table IV

CURRENT INTENSITY IN DIFFERENT STATES FOR VARIOUS PLATFORMS

\begin{tabular}{|c|c|c|c|}
\hline State & STM MS1.0 & OpenMoteSTM & GINA \\
\hline CPU On & $4 m A @ 12 \mathrm{MHz}$ & $7.54 m A @ 16 \mathrm{MHz}$ & $32 m A @ 16 \mathrm{MHz}$ \\
\hline Radio Tx & $4.9 m A$ & $13.7 m A$ & $13.7 m A$ \\
\hline Radio Rx & $4.5 m A$ & $11.6 m A$ & $11.6 m A$ \\
\hline Radio Idle & $1.5 m a$ & - & - \\
\hline Board Off & $0.002 m A$ & $0.4 m A$ & $0.4 m A$ \\
\hline
\end{tabular}

\section{B. Energy Consumption Evaluation for Reserved Slots}

In this section, we use the figures of the current consumption on the STM MS1.0 platform. We consider $V_{B a t}=3 \mathrm{~V}$ and constant.

Table V

ENERGY CONSUMPTION, ONE SLOT

\begin{tabular}{|c|c|c|c|c|}
\hline \multicolumn{2}{|c|}{ Packet Size } & DSME & TSCH (default) & TSCH(optimized) \\
\hline \multirow{2}{*}{30 bytes } & TxSlot & $33.7 \mu \mathrm{J}$ & $61 \mu \mathrm{J}$ & $60 \mu \mathrm{J}$ \\
\cline { 2 - 5 } & RxSlot & $33.6 \mu \mathrm{J}$ & $77.6 \mu \mathrm{J}$ & $54.7 \mu \mathrm{J}$ \\
\hline \multirow{2}{*}{60 bytes } & TxSlot & $59.3 \mu \mathrm{J}$ & $86.6 \mu \mathrm{J}$ & $85.7 \mu \mathrm{J}$ \\
\cline { 2 - 5 } & RxSlot & $58.1 \mu \mathrm{J}$ & $102 \mu \mathrm{J}$ & $79.1 \mu \mathrm{J}$ \\
\hline \multirow{2}{*}{90 bytes } & TxSlot & $85 \mu \mathrm{J}$ & $112.2 \mu \mathrm{J}$ & $111.3 \mu \mathrm{J}$ \\
\cline { 2 - 5 } & RxSlot & $82.5 \mu \mathrm{J}$ & $126.5 \mu \mathrm{J}$ & $103.6 \mu \mathrm{J}$ \\
\hline
\end{tabular}

Table V presents the energy consumption for a DSME GTS slot, and a TSCH Timeslot, based on two slot configurations: the default slot [2], [10] and the slot with reduced Rx Guard Times, based on the work of Stanislowski et al. on adaptive synchronization in a TSCH network [13].

DSME obtains lower energy consumption than TSCH because synchronization before reception in TSCH adds a significant part to the overall energy consumption. Stanislowski et al. proposed a synchronization procedure that would allow keeping the clock drift in a controlled range of $\pm 100 \mu \mathrm{s}$ in an experimental setup [13], which would mean that the average tsR $x W$ ait would be reduced ten times. The procedure would also allow considering shorter synchronization times for ACK reception.

Figure 5 summarizes the breakdown of the energy consumption over different phases during transmission and reception. The data is obtained for a packet size of 30 bytes. For bigger packets, the relative overhead becomes less important. We note that using Enhanced ACKs adds a significant penalty to the energy consumption. DSME is more efficient from this point of view.
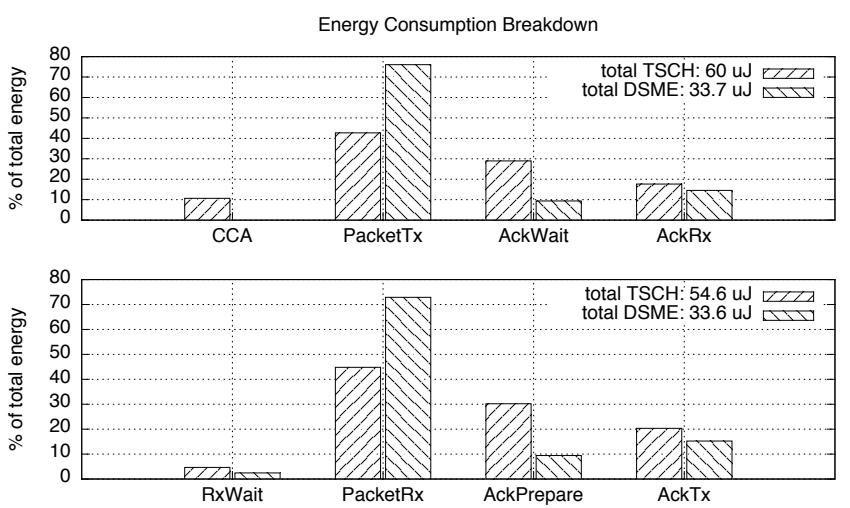

Figure 5. Energy consumption breakdown for different phases

\section{Throughput and Delay}

We evaluate throughput and expected delay for two classes of devices: router nodes and leaves. Leaves need one slot to transmit a packet, whereas routers need two slots: one for receiving a packet and another for forwarding it to another node. In a DSME network, router nodes (coordinators) must wake up for two superframes during a Beacon Interval: one for communicating with their associated devices and another one for forwarding a packet to their own coordinator. We will take into account the two active periods in the superframes for forwarding one packet in the total measurement of throughput.

In a TSCH network, the routing structure does not imply differentiated behaviors inside shared slots. We still need to consider two slots inside a superframe for routers to account for packet forwarding. 


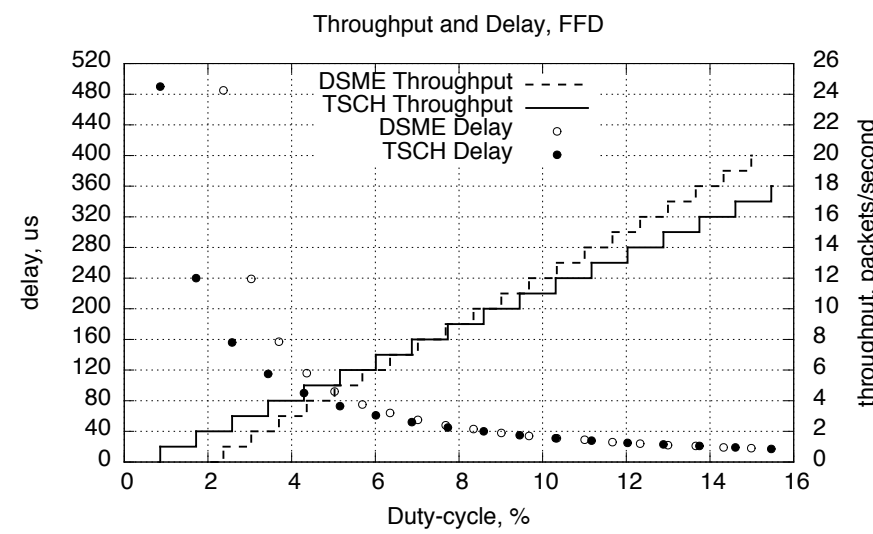

Figure 6. Throughput and delay of routers in function of duty cycle

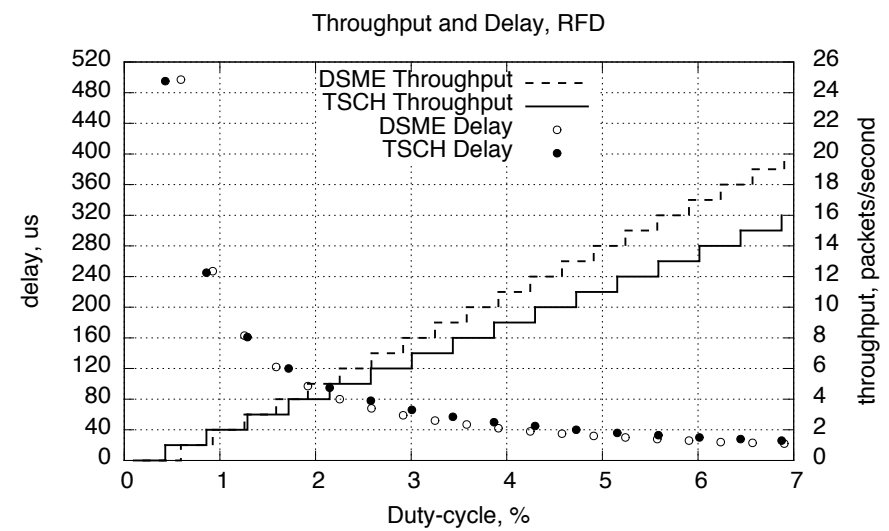

Figure 7. Throughput and delay of leaves in function of duty cycle

For the delay, we consider that active slots are evenly distributed across the measurement period (the Beacon Interval in case of DSME and the slotframe in the case of TSCH), which corresponds to the best case scenario.

Figs. 6 and 7 show total throughput and delay for router and leaf nodes in DSME and TSCH networks for the parameters described in Section III and the duty cycle computed for packets 90 bytes. We can observe that both protocols exhibit similar delays for a given duty cycle, nevertheless, TSCH routers obtain shorter delay and higher throughput for low duty cycles. The CAP in DSME contributes significantly to the duty cycle of the device. As more slots are allocated, DSME benefits from a better slot organization.

Then, we look at lower duty cycles under $1 \%$. For DSME, we increase BeaconOrder to 10 , which gives a BeaconInterval of $15.728 \mathrm{~ms}$, roughly $16 \mathrm{~s}$. For TSCH, we increase the slotframe to 1570 timeslots, or $15700 \mathrm{~ms}$. Neither the Superframe Order nor the Timeslot Length do not change so the analysis concerning the packet transfer in reserved slots is still valid for this second scenario. Figs. 8 and 9 present the results and show similar effects.

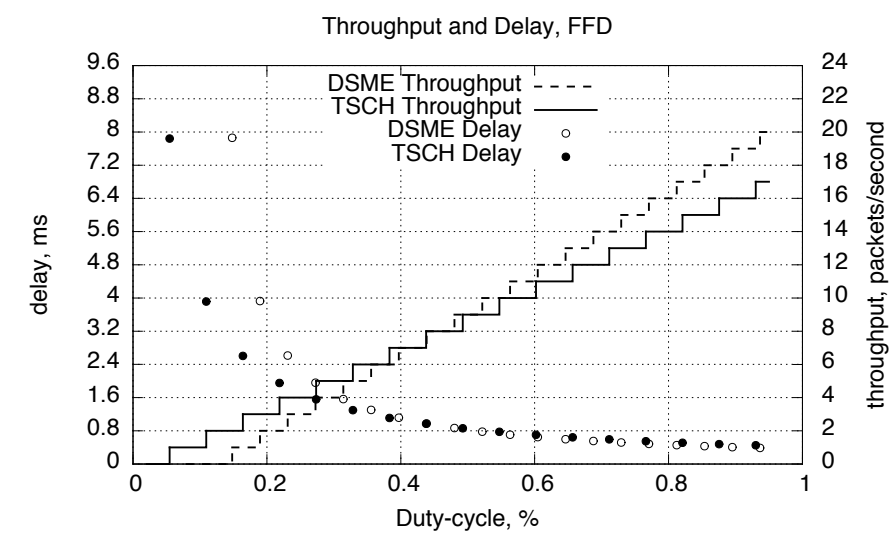

Figure 8. Throughput and delay of routers in function of duty cycle, for low duty cycles

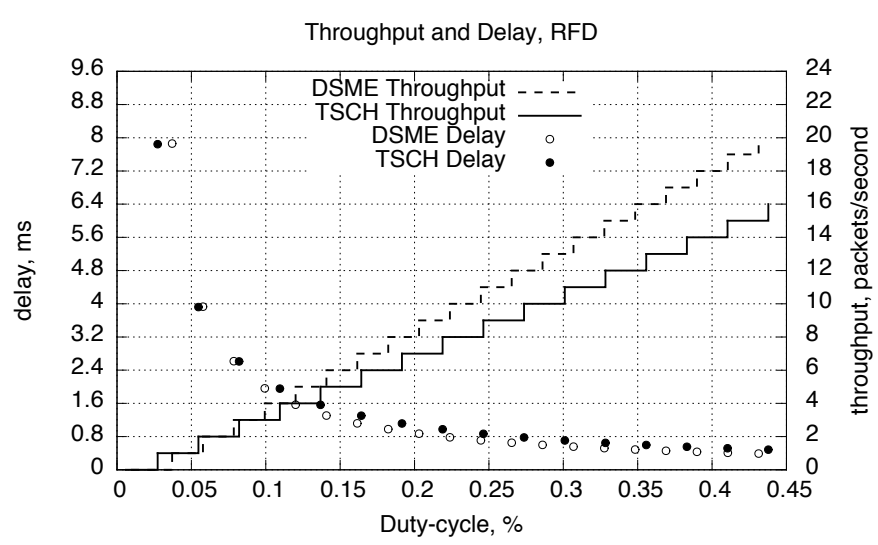

Figure 9. Throughput and delay of leaves in function of duty cycle, for low duty cycles

\section{RELATED WORK}

Shabro et al. considered the applicability of DSME and TSCH [14], among other protocols, to Condition Monitoring Diagnosis in Smart Grid applications in the context of IEC 65850 [15]. It is the only comparison between DSME and TSCH that we are aware of and still, the paper does not provide performance comparisons.

Several authors have analyzed the individual performance of TSCH, DSME, and beacon-enabled 802.15.4. Koubaa et al. applied the network calculus to analyze the limitations of a cluster-tree network of a given structure (width and depth) and transposed the model to a 802.15.4 beaconenabled network [16]. The proposed model can be easily adapted for DSME.

In our previous work, we studied the problem of duty cycle adaptation for an energy harvesting beacon-enabled 802.15.4 network [4]. The choice of the duty cycle relies on a proper combination of $B O-S O$, the beacon and superframe order, since GTS slots in the 802.15.4 beacon-enabled mode can 
only be allocated by the PAN Coordinator. Both DSME and TSCH allow for a finer adjustment of the duty cycle by managing reserved slots.

Tinka et al. proposed a solution for connecting mobile TSCH nodes [7]. Because of the highly instable nature of the network, the TSCH synchronization mechanism is not suited, and instead, nodes rely on a GPS signal that synchronizes all nodes each $1 \mathrm{~s}$. The energy cost of the synchronization is not discussed.

Duquennoy et al. [9] recently proposed a new scheduling algorithm for TSCH. The algorithm separates the traffic in categories (application, routing, MAC) and allocates different schedules for each traffic plane. This approach narrows the gap between TSCH and DSME, since DSME provides a similar separation, for example, with the reserved slots for the Enhanced Beacon emission.

\section{CONCLUSIONS}

The contribution of this paper is twofold: we have presented side-by-side the DSME and TSCH modes introduced in the 802.15.4e standard [2] and compared their respective performance. Under certain configurations, both protocols operate similarly in terms of the medium access: time division multiplexing with channel diversity.

Our analytical results have underpinned a slight advantage of DSME in terms of the energy consumption during data transfers. The reason for this difference is the synchronization method used in TSCH networks. As a consequence, a TSCH network also exhibits worse performance in terms of the total throughput for higher duty cycles. For applications that send little data, the fixed slot configuration of TSCH results in wasted bandwidth. DSME can allocate shorter slots for applications that transmit short packets. With respect to delay, TSCH obtains shorter delay and higher throughput for low duty cycles.

The future work will consist of validating the results by running both protocols on a real platform and comparing other aspects of the network lifecycle: network discovery, scheduling, node mobility, and synchronization.

\section{ACKNOWLEDGEMENTS}

This work was partially supported by the French Ministry of Research project IRIS under contract ANR-11-INFR-016.

\section{REFERENCES}

[1] "IEEE Standard for Information technology- Local and metropolitan area networks- Specific requirements- Part 15.4: Wireless Medium Access Control (MAC) and Physical Layer (PHY) Specifications for Low Rate Wireless Personal Area Networks (WPANs)," IEEE Std 802.15.4-2006 (Revision of IEEE Std 802.15.4-2003), pp. 1-320, Sep. 2006.

[2] "IEEE Standard for Local and metropolitan area networks-Part 15.4: Low-Rate Wireless Personal Area Networks (LR-WPANs) Amendment 1: MAC sublayer,' IEEE Std 802.15.4e-2012 (Amendment to IEEE Std 802.15.4-2011), pp. 1-225, Apr. 2012.

[3] T. Winter, "RPL: IPv6 Routing Protocol for Low-Power and Lossy Networks." [Online]. Available: https://tools.ietf.org/html/rfc6550

[4] L.-O. Varga, G. Romaniello, M. Vucinic, M. Favre, A. Banciu, R. Guizzetti, C. Planat, P. Urard, M. Heusse, F. Rousseau, O. Alphand, E. Dublé, and A. Duda, "GreenNet: an Energy Harvesting IP-enabled Wireless Sensor Network," IEEE IOT Journal, 2015.

[5] M. Vucinic, G. Romaniello, L. Guelorget, B. Tourancheau, F. Rousseau, O. Alphand, A. Duda, and L. Damon, "Topology construction in RPL networks over beacon-enabled 802.15.4," in 2014 IEEE Symposium on Computers and Communication (ISCC), Jun. 2014, pp. 1-7.

[6] M. Palattella, N. Accettura, M. Dohler, L. Grieco, and G. Boggia, "Traffic Aware Scheduling Algorithm for reliable low-power multihop IEEE 802.15.4e networks," in 2012 IEEE 23rd International Symposium on Personal Indoor and Mobile Radio Communications (PIMRC), Sep. 2012, pp. 327-332.

[7] A. Tinka, T. Watteyne, and K. Pister, "A Decentralized Scheduling Algorithm for Time Synchronized Channel Hopping." Springer Berlin Heidelberg, Aug. 2010, pp. 201-216. [Online]. Available: http://link.springer.com/chapter/10.1007/978-3-642-17994-5_14

[8] N. Accettura, M. Palattella, G. Boggia, L. Grieco, and $\bar{M}$. Dohler, "Decentralized Traffic Aware Scheduling for multi-hop Low power Lossy Networks in the Internet of Things," in World of Wireless, Mobile and Multimedia Networks (WoWMoM), 2013 IEEE 14th International Symposium and Workshops on a, Jun. 2013, pp. 1-6.

[9] S. Duquennoy, B. A. Nahas, O. Landsiedel, and T. Watteyne, "Orchestra: Robust Mesh Networks Through Autonomously Scheduled TSCH," in Proceedings of the 13th ACM Conference on Embedded Networked Sensor Systems, SenSys 2015, Seoul, South Korea, November 1-4, 2015, 2015, pp. 337-350.

[10] X. Vilajosana and K. Pister, "Minimal 6tisch Configuration." [Online]. Available: https://tools.ietf.org/html/draft-ietf-6tisch-minimal-06

[11] Z. Shelby, K. Hartke, and C. Bormann, "The Constrained Application Protocol (CoAP)." [Online]. Available: https://tools.ietf.org/html/rfc7252

[12] X. Vilajosana, Q. Wang, F. Chraim, T. Watteyne, T. Chang, and K. Pister, "A Realistic Energy Consumption Model for TSCH Networks," IEEE Sensors Journal, vol. 14, no. 2, pp. 482-489, Feb. 2014.

[13] D. Stanislowski, X. Vilajosana, Q. Wang, T. Watteyne, and K. Pister, "Adaptive Synchronization in IEEE802.15.4e Networks," IEEE Transactions on Industrial Informatics, vol. 10, no. 1, pp. 795-802, Feb. 2014.

[14] M. Shabro and S. Ali Ghorashi, "Comparison of IWSN MAC Protocols for IEC 61850 Applications," International Journal of Innovative Research in Electrical, Electronics, Instrumentation and Control Engineering, vol. 3, no. 6, Jun. 2015.

[15] Siemens, "IEC 61850 standard." [Online]. Available: http://www.energy.siemens.com

[16] A. Koubaa, M. Alves, and E. Tovar, "Modeling and Worst-Case Dimensioning of Cluster-Tree Wireless Sensor Networks." IEEE, 2006, pp. 412-421. 